\title{
01.3
}

\section{Коллективная динамика ротаторов, связанных общим импульсным полем}

\author{
(C) М.И. Болотов, Г.В. Осипов
}

Национальный исследовательский Нижегородский государственный университет им. Н.И. Лобачевского, Нижний Новгород

E-mail: bolotov_maxim_ilich@mail.ru

Поступило в Редакцию 19 мая 2016 г.

Исследуется коллективная динамика в ансамбле активных ротаторов, связанных общим импульсным полем. Проводится численное изучение динамики ансамбля при различных значениях параметров системы. На основе полученных данных в зависимости от значения параметра синхронности делается вывод о коллективном поведении элементов ансамбля, характере эволюции поля ансамбля, зависимости поведения поля ансамбля от синхронности элементов ансамбля.

Под синхронизацией обычно понимается процесс достижения связанными объектами единого ритма функционирования. Синхронизация - это очень распространенное явление в природе и технике [1-3]. Целью данной работы является исследование синхронных режимов в ансамбле ротаторов, связанных общим полем. Уравнения, описывающие динамику элементов данного ансамбля, являются предельным случаем связанных маятниковых уравнений, которые описывают многие физические системы.

Рассмотрим ансамбль связанных идентичных математических маятников в вязкой среде. Динамика каждого маятника задается уравнением

$$
\ddot{\varphi}_{i}+\lambda \dot{\varphi}_{i}+\sin \varphi_{i}=\gamma+G_{i}(t, \varphi, \dot{\varphi}),
$$

где $i=1,2, \ldots, N, \varphi_{i}-$ фаза $i$-го маятника, $\varphi=\left(\varphi_{1}, \varphi_{2}, \ldots, \varphi_{N}\right)$, $\lambda \geqslant 0-$ коэффициент диссипации, $\gamma \geqslant 0-$ момент постоянной внешней силы, $G_{i}(t, \varphi, \dot{\varphi})-$ связь $i$-го маятника с другими маятниками. В случае $G_{i}(t, \varphi, \dot{\varphi})=0$ система (1) представляет собой систему не связанных маятников под действием момента постоянной внешней силы [1], при этом каждое уравнение системы (1), например, может описывать динамику контакта Джозефсона [1]. 
Связанные маятники являются одним из примеров моделей, которые могут быть использованы для исследования различных коллективных явлений в сосредоточенных и пространственно-распределенных системах. В приложениях связь между элементами может быть разной. В случае связи $G_{i}(t, \varphi, \dot{\varphi})=g \sin \left(\varphi_{i-1}-\varphi_{i}\right)+g \sin \left(\varphi_{i+1}-\varphi_{i}\right)$, где $g$ сила связи, система (1) представляет собой цепочку диффузионно связанных маятников. Эта модель используется для изучения различных эффектов в ансамблях джозефсоновских контактов [4], магнитной цепи Гейзенберга [5], системах фазовой автоподстройки [6], активных антенных решетках [6]. Если $G_{i}(t, \varphi, \dot{\varphi})=\frac{g}{N} \sum_{j=1}^{N} \sin \left(\varphi_{j}-\varphi_{i}\right)$, где $g-$ сила связи, то (1) представляет собой модель Курамото с инерцией. Модель Курамото является универсальной для большого числа приложений, например связанных полупроводниковых лазеров [7], ансамблей СВЧ-генераторов [8] и др.

Возможным вариантом связи в задаче достижения синхронных режимов является связь элементов ансамбля через общее поле. В этом случае $G_{i}(t, \varphi, \dot{\varphi})=g E(t)$, где $g$ - сила связи, $E(t)$ - динамическая переменная состояния общего поля.

Рассмотрим ансамбль, который состоит из $N$ идентичных маятников, каждый из которых связан через общее поле со всеми другими маятниками. Произведем замену времени $\tau \lambda=t$ и предположим, что среда обладает сильной вязкостью $(\lambda \gg 1)$, тогда движения в системе можно разделить на быстрые и медленные [9]. При этом устойчивые медленные движения каждого маятника подчиняются уравнению

$$
\dot{\varphi}_{i}=\gamma-\sin \varphi_{i}+g E(t) .
$$

Уравнение (2) моделирует поведение ансамбля активных ротаторов [10-14], где состояние $i$-го ротатора ансамбля задается переменной $\varphi_{i}$, которая принимает значения из отрезка $[0 ; 2 \pi]$. В качестве модели динамики общего поля одной из наиболее распространенных является модель поля, формируемого импульсами, поступающими от всех элементов ансамбля. Изучение синхронных режимов в подобных ансамблях представляет большой интерес исследователей, как физиков, так и математиков [15-19].

Уравнения (2) определяют состояния ротаторов на отрезке $[0 ; 2 \pi]$. Как только $\varphi_{i}$ достигает значения $2 \pi$, с $i$-го осциллятора в уравнение

Письма в ЖТФ, 2016, том 42, вып. 23 
для общего поля подается импульсное воздействие. Эволюция поля ансамбля при этом задается уравнением

$$
\ddot{E}+2 \alpha \dot{E}+\alpha^{2} E=\beta \sum_{n \mid t_{n}<t} \delta\left(t-t_{n}\right),
$$

где $\alpha>0$ - параметр эволюции, характеризующий затухание поля на межимпульсном интервале, $\beta>0$ - параметр, задающий величину импульсного воздействия, $t_{n}-$ момент времени поступления $n$-го импульса.

Введем следующую переменную $Q=\alpha E+\dot{E}$. При интегрировании уравнения (3) от момента времени $t_{n}$ до момента $t<t_{n+1}$ получается

$$
\begin{gathered}
E(t)=\left(E\left(t_{n}\right)+\left(t-t_{n}\right) Q\left(t_{n}\right)\right) e^{-\alpha\left(t-t_{n}\right)}, \\
Q(t)=Q\left(t_{n}\right) e^{-\alpha\left(t-t_{n}\right)} .
\end{gathered}
$$

В момент времени $t_{n+1}$ значение переменной $Q(t)$ определяется следующим образом:

$$
Q\left(t_{n+1}\right)=Q\left(t_{n}\right) e^{-\alpha\left(t_{n+1}-t_{n}\right)}+\beta,
$$

где первое слагаемое отвечает за затухание поля $E$, а второе слагаемое - за величину внешнего импульсного воздействия, т. е. в момент времени поступления импульса производная общего поля $\dot{E}(t)$ получает импульсную добавку, амплитуда которой равна $\beta$. При этом общее поле $E(t)$ является непрерывной величиной.

Таким образом, рассматривается система ротаторов, на каждый из которых подается внешнее воздействие, которое определяется текущим состоянием всех ротаторов.

Будем считать динамику элементов ансамбля синхронной, если разность между переменными состояния индивидуальных осцилляторов мала, т. е. $\varphi_{1} \approx \varphi_{2} \approx \cdots \approx \varphi_{N}$. Введем параметр синхронности $\mu$ следующим образом:

$$
\mu=1-\frac{\sum_{i, j} \rho\left(\varphi_{i}-\varphi_{j}\right)}{N \sum_{i} \rho\left(\varphi_{i}^{l}-\varphi_{1}^{l}\right)},
$$

где $\rho\left(\varphi_{i}, \varphi_{j}\right)=\left|\varphi_{i}-\varphi_{j}\right| \bmod 2 \pi-$ расстояние между $\varphi_{i}$ и $\varphi_{j}$ на окружности $[0 ; 2 \pi], \varphi_{i}-$ состояние $i$-го осциллятора в фиксированный момент времени, $\varphi_{i}^{l}-$ состояние $i$-го осциллятора, соответствующее

Письма в ЖТФ, 2016, том 42, вып. 23 


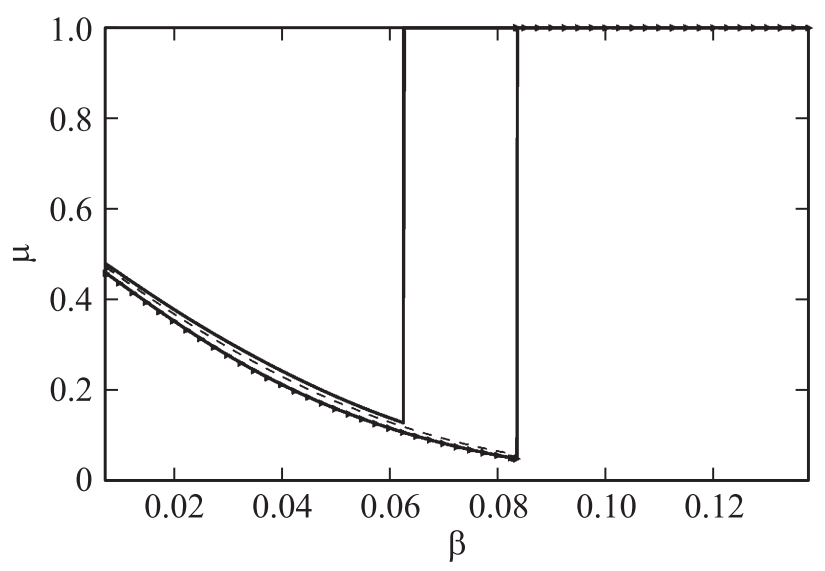

Рис. 1. Значения параметра синхронности $\mu$ для режимов с наименьшим (линия с маркерами), некоторым промежуточным (штриховая линия) и наибольшим (сплошная линия) значением параметра $\mu$ при $N=100, \gamma=1.03, g=1.0$, $\alpha=1.5$ в зависимости от $\beta$.

линейному распределению фаз на $[0 ; 2 \pi]$. Данный параметр синхронности выбран таким образом, чтобы линейному распределению фаз ротаторов на отрезке $[0 ; 2 \pi]$ (splay state), т.е. состоянию, при котором среднее расстояние от фазы каждого ротатора до фазы другого ротатора было наибольшим, соответствовало наименьшее значение параметра синхронности (в данном случае $\mu=0$ ), а состоянию, когда фазы всех ротаторов совпадают, наибольшему значению параметра синхронности $(\mu=1)$. Таким образом, $\mu=0$ соответствует полностью асинхронному режиму, а $\mu=1-$ полностью синхронному.

Исследуем свойства синхронности поведения ротаторов в зависимости от величины параметра импульсного воздействия $\beta$. Зафиксируем значения параметров $N=100, \gamma=1.03, g=1.0, \alpha=1.5$. При $\gamma=1.03$ правая часть уравнения (2) принимает малые значения, когда фаза ротатора $\varphi_{i}$ близка к $\frac{\pi}{2}$. Следовательно, фаза каждого осциллятора проводит долгое время в окрестности $\frac{\pi}{2}$.

На динамику ансамбля существенно влияет начальное распределение фаз ротаторов. Будем рассматривать случай линейного распреде-

Письма в ЖТФ, 2016, том 42, вып. 23 


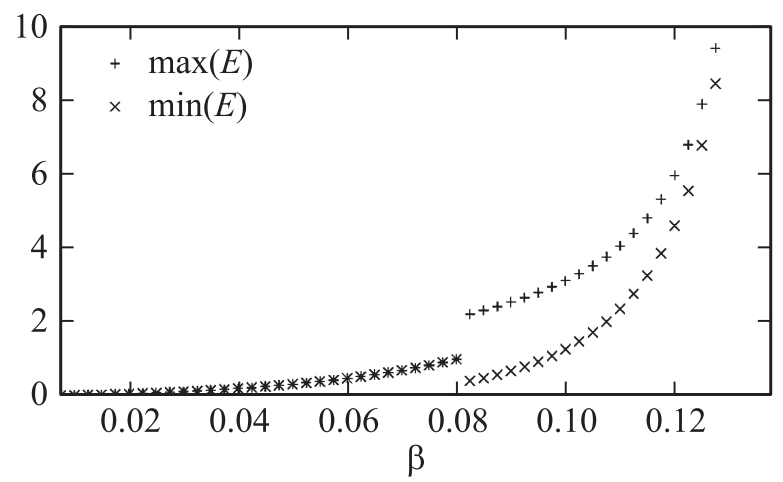

Рис. 2. Переход от асинхронной динамики к синхронной в зависимости от величины импульсного воздействия $\beta$ при $N=100, \gamma=1.03, g=1.0, \alpha=1.5$. Значения локальных максимумов и минимумов поля $E$, соответствующие режиму с наименьшим значением параметра синхронности.

ления фаз на отрезке $\left[0 ; \varphi_{\max }^{0}\right]$ в начальный момент времени. Тогда при малых значениях параметра импульсного воздействия $\beta<0.0625$ каждому начальному распределению фаз однозначно соответствует свой устойчивый асинхронный режим динамики, синхронный режим при этом является неустойчивым. Асинхронному режиму с наименьшими значениями параметра синхронности соответствует поле, значение которого близко к постоянному с малыми отклонениями, другим асинхронным режимам - поле периодическое с малой амплитудой. При больших значениях $\beta(\beta>0.0625)$ синхронный режим становится устойчивым, при этом ансамбль эволюционирует к синхронному режиму для всех $0 \leqslant \varphi_{\max }^{0} \leqslant \varphi_{s}^{0}$. При $\varphi_{\max }^{0}>\varphi_{s}^{0}$ динамика ансамбля асинхронная, и каждому начальному распределению фаз соответствует свой устойчивый режим. При $\beta>0.0836$ ансамбль эволюционирует к полностью синхронному режиму для любого начального распределения фаз. Поле ансамбля в случае синхронной динамики периодическое с большой амплитудой. Обнаружено, что при переходе к синхронному режиму наблюдается гистерезис. На рис. 1 представлены значения параметра синхронности в зависимости от силы импульсного воздействия $\beta$.

При дальнейшем увеличении значения величины внешнего воздействия $\beta$ происходит постепенное увеличение среднего значения

Письма в ЖТФ, 2016, том 42, вып. 23 
поля $E$, поле при этом остается периодическим, элементы ансамбля совершают синхронные вращения. При приближении к критическому значению $\beta=0.14$ среднее значение поля начинает резко увеличиваться (рис. 2). При достижении параметром $\beta$ критического значения наступает ситуация, когда поле $E$ резко растет во времени, при этом импульс, прикладываемый к полю (получаемый при достижении фазы некоторого ротатора значения $2 \pi$ ), дает прибавку к полю больше, чем убывает значение поля в межимпульсный интервал из-за затухания (второе слагаемое в уравнении (4) больше первого слагаемого, т.е. величина $Q$, а следовательно, и величина общего поля $E$ растут со временем) при $\beta>0.14$, т.е. затухание не успевает компенсировать внешнего воздействия на поле. Вращения элементов ансамбля при этом не синхронизованы. Рост общего поля $E$ приводит к росту частоты вращений каждого ротатора, что в свою очередь ведет к дальнейшему росту поля.

Данный эффект можно объяснить следующим образом. При $\gamma>1$ уравнение (2) можно усреднить за период $2 \pi$. После усреднения $\sin \phi_{i}=0$ и уравнение приобретает вид $\dot{\phi}=\gamma+g E$, где в рассматриваемом приближении $\dot{\phi}$ представляет собой усредненную за период фазовую скорость ротатора. Из этого уравнения видно, что при увеличении поля $E$ усредненная фазовая скорость растет, а значит, растет и частота вращения ротатора, что и требовалось доказать.

В результате численного исследования динамики ансамбля было установлено существование мультистабильности в системе, в частности имеет место множество асинхронных режимов, когда элементы ансамбля асинхронны, а среднее поле либо постоянное с малыми возмущениями, либо периодическое с малой амплитудой; синхронного режима, при котором элементы ансамбля полностью синхронны, а среднее поле периодическое с большой амплитудой, а также режима, когда рост общего поля приводит к росту частоты каждого ротатора.

Авторы выражают искреннюю благодарность А.С. Пиковскому за полезные обсуждения и замечания в ходе работы. Численные эксперименты проводились на суперкомпьютере „Лобачевский“ Нижегородского государственного университета им. Н.И. Лобачевского. Работа была выполнена при поддержке Российского научного фонда (проект № 1412-00811).

3 Письма в ЖТФ, 2016, том 42, вып. 23 


\section{Список литературы}

[1] Pikovsky A., Rosenblum M., Kurths J. Synchronization: a universal concept in nonlinear sciences. Cambridge: Cambridge University Press, 2001.

[2] Osipov G., Kurths J., Zhou Ch. Synchronization in oscillatory networks. Berlin, Heidelberg: Springer, 2007.

[3] Mosekilde E., Maiistrenko Yu., Postnov D. Chaotic synchronization. Application to living systems. Singapore: World Scientific, 2002.

[4] Wiesenfeldt K., Colet P., Strogatz S.H. // Phys. Rev. Lett. 1996. V. 76. P. 404407.

[5] Wang X.Y., Taylor P.L. // Phys. Rev. Lett. 1996. V. 76. P. 640.

[6] Матросов В.В., Шалфеев В.Д. Нелинейная динамика систем фазовой синхронизации. Нижний Новгород: Изд-во Нижегородского госуниверситета, 2013.

[7] Kozyrev G., Vladimirov A.G., Mandel P. // Phys. Rev. Lett. 2000. V. 85. N 18. P. 3809.

[8] York R.A., Compton R.C. // IEEE Transactions on Automatic Control. 2012. V. 57. N 4. P. 920.

[9] Анищенко В.С., Астахов В.В., Вадивасова Т.Е. Регулярные и хаотические автоколебания. Синхронизация и влияние флуктуаций. М.: Изд-во „Интеллект", 2009.

[10] Sakaguchi H. // J. Korean Phys. Soc. 2008. V. 53. N 2. P. 1257.

[11] Kanamaru T., Sekine M. // Phys. Rev. E. 2003. V. 67. P. 031916.

[12] Komin N., Toral R. // Phys. Rev. E. 2010. V. 82. P. 051127.

[13] Daido H. // Phys. Rev. E. 2015. V. 91. P. 012925.

[14] Ionita F., Meyer-Ortmanns H. // Phys. Rev. E. 2014. V. 112. P. 094101.

[15] Allene C., Cattani A., Ackman J.B. et al. // J. Neurosci. 2008. V. 28. P. 12851.

[16] Abbott L.F., Van Vreeswijk C. // Phys. Rev. E. 1993. V. 48. P. 1483.

[17] Mohanty P.K., Politi A. // J. Phys. A. 2006. V. 39. P. L415.

[18] Olmi S., Livi R., Politi A., Torcini A. // Phys. Rev. E. 2010. V. 81. P. 046-119.

[19] Zillmer R., Livi R., Politi A., Torcini A. //. Phys. Rev. E. 2007. V. 76. P. 046102. 